\title{
Tumor associated macrophages and its nanovesicles: Immunological insights in cerebral glioma
}

\author{
Turano $\mathrm{E}^{1}$, Farinazzo $\mathrm{A}^{1}$, El Mously $\mathrm{S}^{* 2}$, Calabria $\mathrm{F}^{1}$, Jugerson $\mathrm{I}^{1}$, Bonetti $\mathrm{B}^{1}$, Bazzoli $\mathrm{E}^{1}$ \\ ${ }^{1}$ Neuroscience Department, Faculty of Medicine, Verona University, Italy \\ ${ }^{2}$ Neurology Department, Faculty of Medicine, Fayoum University, Egypt
}

Received: December 2, 2019

Accepted: December 20, 2019 Online Published: February 10, 2020

DOI: $10.5430 /$ jst.v10n1p14

URL: https://doi.org/10.5430/jst.v10n1p14

\begin{abstract}
Purpose: The immune system has a key role in glioma progression, especially the tumor associated macrophages (TAMs). In-vivo, we aimed to study the total TAMs and differential M1 and M2 TAM infiltration in low grade (LGG) versus high grade gliomas (HGG). Also, we investigated the implication of total TAMs and differential M1 and M2 TAMs infiltration on glioma progression. In-vitro, we studied the effect of soluble factors present in nanovesicles (NV) released from M1 TAMs on the fate of glioma cells.

Methods: In-vivo, we performed immunohistochemistry using iNOS and CD163 (markers for M1 and M2 respectively). In-vitro, we polarized the human monocytes U937 cell line into M1, we isolated the NV from the M1-conditioned medium (CM) by centrifugation and filtration; then, the protein content of the NV was quantified by the protein assay. We added M1-NV on U251 glioma cells and we studied the cellular activation of glioma cells using the MTT assay. To assess the apoptosis of U251, we used the flow-cytometry. Apoptotic cells were identified by annexin V and Propidium Iodide (markers for early and late apoptosis respectively).

Results: in-vivo, there is an M1/M2 imbalance in early stages of glioma which is associated with earlier progression to high malignancy. Also, the higher M2 infiltration, the earlier is the progression. In-vitro, M1-NV had a more potent anti-tumor effect compared to its corresponding CM. We assume that our experimental results can be a future treatment for the cerebral glioma.
\end{abstract}

Key Words: Glioma, HGG, LGG, TAMs, Microenvironment

\footnotetext{
${ }^{*}$ Correspondence: El Mously S; Email: smm02@fayoum.edu.eg; Address: Faculty of Medicine, Fayoum University, Keman Fares area, Fayoum, Egypt. 